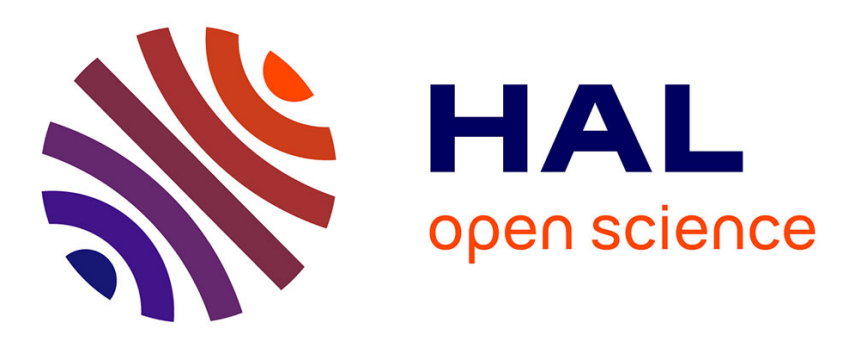

\title{
Bonne reproductibilité dans une population étudiante d'un court questionnaire (14-item Food Frequency Questionnaire) évaluant l'alimentation à risque vasculaire
}

L Balquet, B Noury-Desvaux, V Jaquinandi, Guillaume Mahé

\section{To cite this version:}

L Balquet, B Noury-Desvaux, V Jaquinandi, Guillaume Mahé. Bonne reproductibilité dans une population étudiante d'un court questionnaire (14-item Food Frequency Questionnaire) évaluant l'alimentation à risque vasculaire. Journal des Maladies Vasculaires, 2015, 40 (1), pp.18-23. 10.1016/j.jmv.2014.12.005 . hal-01107843

HAL Id: hal-01107843

https://hal-univ-rennes1.archives-ouvertes.fr/hal-01107843

Submitted on 21 Jan 2015

HAL is a multi-disciplinary open access archive for the deposit and dissemination of scientific research documents, whether they are published or not. The documents may come from teaching and research institutions in France or abroad, or from public or private research centers.
L'archive ouverte pluridisciplinaire HAL, est destinée au dépôt et à la diffusion de documents scientifiques de niveau recherche, publiés ou non, émanant des établissements d'enseignement et de recherche français ou étrangers, des laboratoires publics ou privés. 
Titre : Bonne reproductibilité dans une population étudiante d'un court questionnaire (14item Food-Frequency Questionnaire) évaluant l'alimentation à risque vasculaire.

\section{Auteurs}

Lucie BALQUET $(\mathrm{MSc})^{1}$

Bénédicte NOURY-DESVAUX (Ph.D.) ${ }^{1}$

Vincent JAQUINANDI (MD, Ph.D.) ${ }^{2}$

Guillaume MAHE (MD, Ph.D.) $)^{3,4}$

\section{Affiliations}

${ }^{1}$ APCoSS, Institut de formation en éducation physique et en sport d'Angers (IFEPSA), Université catholique de l'Ouest (UCO). (49, rue des Perrins. Les Ponts de Cé, F-49136. FRANCE).

2 Cabinet de Médecine vasculaire, Trélazé, FRANCE (6, Rue de Bellinière, 49800)

${ }^{3}$ Inserm Centre d'investigation Clinique CIC 1414, Rennes, FRANCE. (2, Rue Henri Le Guilloux, 35033 Rennes, Cedex 9)

${ }^{4}$ Université de Rennes. FRANCE. (2, Rue Henri Le Guilloux, 35033 Rennes, Cedex 9)

Auteur correspondant : Guillaume Mahé. Imagerie Coeur-Vaisseaux. Hôpital Pontchaillou. 2 rue Henri le Guilloux Cedex 09, F-359043. FRANCE. ్ㅛㅛ +33(0)2.99.28.43.21. E-mail: guillaume.mahe@chu-rennes.fr 
TITRE : Bonne reproductibilité dans une population étudiante d'un court questionnaire (14item Food-Frequency Questionnaire) évaluant l'alimentation à risque vasculaire.

Résumé : L'alimentation est un facteur de risque modifiable d'athérosclérose. Un court questionnaire de 14 questions évaluant l'alimentation à risque vasculaire et préalablement validé chez des patients présentant une maladie athéromateuse a été utilisé. La reproductibilité de ce questionnaire n'est pas connue chez les étudiants, population qui serait intéressante à évaluer sur le plan alimentaire.

Méthodes : Ce court questionnaire permet d'évaluer le Score Diététique Vasculaire (SDV) et des scores pour différents groupes alimentaires ayant un rôle dans les maladies athéromateuses.

La reproductibilité a été évaluée chez des étudiants en Licence Sciences et Techniques des Activités Physiques et Sportives (STAPS). Des tests de comparaison de moyennes ont été utilisés entre la mesure 1 et la mesure 2 des scores nutritionnels (délai minimum de 7 jours) et les coefficients intra-classe (ICC) ont été calculés.

Résultats : Trente étudiants (50\% d'hommes) inscrits en Licence STAPS ont été inclus. Cinquante pour cent étaient des hommes et l'indice de masse corporelle moyen était de 21,66 $+/-2,06 \mathrm{~kg} / \mathrm{m}^{2}$. Le délai moyen entre le remplissage des 2 questionnaires était de $19+/-9$ jours. Le SDV moyen était de $0,50+/-3,70$ lors de la première mesure et de $0,30+/-3,14$ lors de la deuxième mesure (Non significatif). Aucun score de groupe alimentaire n'était significativement différent entre la première et la deuxième mesure. L'ICC du SDV était de $0,68$ [intervalle de confiance à $95 \%: 0,43-0,83]$.

Conclusion : Ce questionnaire qui permet d'évaluer l'alimentation à risque vasculaire possède une reproductibilité satisfaisante. Cet outil peut être utile pour étudier l'alimentation à risque vasculaire de larges populations étudiantes.

Mots-clés : questionnaire, athérosclérose, reproductibilité, méthodes, maladie vasculaire, 
régime, nutrition, facteur de risque cardiovasculaire

Title: Good reproducibility of a 14-item food frequency questionnaire for cardiovascular prevention in students.

\begin{abstract}
:
Diet is a modifiable risk factor of atherosclerosis. A 14-item food-frequency questionnaire (FFQ) has been developed. The reproducibility of this FFQ is unknown in a student population whereas its use could be of interest.
\end{abstract}

Methods: This FFQ allows calculating different scores for different food groups involved in cardiovascular disease. The vascular dietary score (SDV) can be calculated. The SDV ranges from -17 to +19 . The higher the SDV, the better diet. Reproducibility was assessed in Sport faculty students using mean tests comparing measurement 1 and 2 (minimum time interval $\geq$ 7 days) and intra-class correlation (ICC) tests.

Results : Thirty students (50\% men) were included in a French Sports Faculty. Time between two FFQ assessments was $19+/-9$ days. Mean SDV was $0.50+/-3.70$ for the first assessment and $0.30+/-3.14$ for the second one (non significant). Any score for each food group was statistically significant between the first and the second measurement. ICC of SDV was 0.68 [95\% confidence interval: $0.43-0.83]$.

Conclusion: This FFQ that assesses a risky vascular diet has good reproducibility. This tool could be useful for large studies involving students.

Key-words: Food frequency Questionnaire, atherosclerosis, diet, reproducibility, vascular disease, nutrition, cardiovascular risk factor 


\section{INTRODUCTION}

Les maladies cardiovasculaires représentent un enjeu économique très important dans le domaine de la santé publique. Plusieurs études ont montré que l'alimentation et les habitudes de vie, telles que le niveau d'activité physique, représentent des facteurs de risque modifiables dans les maladies cardiovasculaires notamment liées à l'athérosclérose . Lancé en 2001, le Programme National Nutrition Santé (PNNS) est un plan de santé publique visant à améliorer l'état de santé de la population en agissant sur l'un de ses déterminants majeurs : la nutrition. Pour le PNNS, la nutrition s'entend comme l'équilibre entre les apports liés à l'alimentation et les dépenses occasionnées par l'activité physique (http://www.mangerbouger.fr/pnns/). Les actions mises en œuvre par le PNNS ont comme finalité de promouvoir, dans l'alimentation et le mode de vie, les facteurs de protection de la santé et de réduire l'exposition aux facteurs de risque de maladies chroniques-Déterminants pour la santé, l'alimentation et l'activité physique ont été intégrées aux sept paramètres de la «santé cardiovasculaire idéale » définie par l'American Heart Association en 2010 .

Un questionnaire d'évaluation de l'alimentation à risque vasculaire composé de 14 questions a été développé et comparé à une enquête alimentaire sur 7 jours et à des marqueurs biologiques. Ce questionnaire a été utilisé dans plusieurs populations présentant des pathologies athéromateuses (infarctus du myocarde, accident vasculaire cérébral ischémique et artériopathie oblitérante des membres inférieurs). Lors de l'utilisation de celui-ci chez des patients âgés en moyenne de 50 ans, un score nutritionnel faible avait été retrouvé chez les patients présentant une maladie athéromateuse . Il a aussi été utilisé en cabinet de médecine générale (250 patients) montrant sa facilité d'utilisation à grande échelle .

La prévention promue par le PNNS s'adresse à l'ensemble de la population mais son intérêt apparaît d'autant plus important chez les jeunes. En effet, les habitudes de vie sont souvent prises dans la première partie de vie et l'effet de l'alimentation étant liée à la durée 
d'exposition, il apparaît intéressant de pouvoir évaluer l'alimentation à risque vasculaire chez les étudiants pour pouvoir juger de la qualité alimentaire des étudiants et pouvoir agir en fonction. Cependant, avant la réalisation d'études de grande ampleur chez des étudiants, il convient d'étudier la reproductibilité de ce type de questionnaire dont la reproductibilité n'a été étudiée que chez des patients âgés en moyenne de 58 ans . Dans cette étude la reproductibilité étudiée chez 20 patients était excellente . L'objectif de ce travail est d'étudier la reproductibilité d'un court questionnaire évaluant l'alimentation à risque vasculaire dans une population étudiante.

\section{MATERIELS ET METHODES}

Population :

Les sujets ont tous été recrutés au sein de l'Institut de Formation en Education Physique et en Sport d'Angers (IFEPSA), sur une période de trois semaines. Ils devaient être volontaires et être inscrits en Licence au sein de cet établissement pour être inclus dans l'étude. Les participants étaient éligibles s'ils avaient plus de 18 ans. Tous les participants ont signé un consentement éclairé.

Questionnaire alimentaire (14-item Food frequency questionnaire, FFQ) à risque vasculaire :

Contenant quatorze questions, le FFQ permet de déterminer des scores sur les groupes d'aliments jouant un rôle soit néfaste, soit protecteur pour le système vasculaire . Parmi les aliments et nutriments explorés, on distingue les Acides Gras Saturés (AGS), les Acides Gras Mono-Insaturés (AGMI), les Acides Gras Poly-Insaturés oméga 3 (AGPI $\Omega$-3) et les fruits et légumes (F-L). 
Le FFQ présente un groupe de six questions évaluant les AGS provenant du fromage, de la viande rouge, de la charcuterie, des pizzas et tartes salées, des pâtisseries et viennoiseries, et du beurre (questions 1, 2, 4, 5, 7 et 12). Cinq autres questions étudient les AGMI d'origine végétale, les AGPI oméga 3 provenant des poissons (question 3), des noix (question 9), et des graisses végétales (questions 6,13 et 14). Trois questions sont consacrées aux apports en fruits et légumes (questions 8, 10 et 11). Le poids de chaque item dans le questionnaire a été déterminé en utilisant l'enquête alimentaire INCA réalisée en France entre 1994 et 1999.

À chaque groupe alimentaire est attribué un score déterminé à partir d'une grille d'évaluation. Un score négatif, compris entre 0 et -17 , est attribué à la consommation d'AGS. Ce score est négatif en raison de l'influence néfaste des AGS sur les maladies athéromateuses. Un score positif, entre 0 et +19 , correspond à la somme des consommations des AGMI, des AGPI oméga 3 et des F-L. Contrairement aux AGS, ces différents groupes d'aliments sont favorables à la protection vasculaire. L'échelle des AGMI est comprise entre 0 et +4 . Le score des AGPI s'étend de 0 à +8 points. Quant aux F-L, l'échelle s'apprécie de 0 à +7 sachant qu'un score de +7 équivaut à une consommation de cinq F-L par jour.

À partir du score des différents groupes, le FFQ présente l'avantage d'obtenir un score global d'alimentation à risque vasculaire appelé « Score Diététique Vasculaire » (SDV) compris entre -17 et +19 et obtenu par le calcul suivant $:$ SDV $=$ Score protecteur - Score néfaste $=$ $(\mathrm{F}-\mathrm{L}+\mathrm{AGMI}+$ AGPI oméga 3) - (AGS)

Plus le SDV est élevé, c'est-à-dire proche de +19 , plus le régime alimentaire est à faible risque cardiovasculaire. Inversement, l'alimentation est à risque cardiovasculaire lorsque le SDV est proche de -17 . 
Déroulement de l'étude :

Après une information sur l'étude en amphithéâtre avec l'autorisation du directeur de l'IFEPSA, les étudiants souhaitant réaliser l'étude venaient à une première visite, lors de laquelle les caractéristiques générales de sujets étaient enregistrées et les étudiants remplissaient le questionnaire nutritionnel ( $1^{\text {ère }}$ mesure). Au moins 7 jours après lors d'une autre visite, le questionnaire nutritionnel était rempli une seconde fois ( $2^{\text {ème }}$ mesure).

Statistiques :

Les résultats sont exprimés en moyenne +/- écart-type. Les comparaisons de moyenne ont été réalisées avec le test de Mann et Withney. L'erreur typique de mesure a été calculée à partir de la méthode de Hopkins . Celle-ci est obtenue en divisant la déviation standard des différences appariées par racine de 2 . La reproductibilité a été étudiée par le coefficient de corrélation intra-classe (ICC). La valeur d'ICC est comprise entre 0 et 1. Plus l'ICC est proche de 1 plus la reproductibilité est bonne. Une ICC strictement supérieure à 0,75 définie une excellente reproductibilité alors qu'une ICC comprise entre 0,60 et 0,75 est définie une bonne reproductibilité . L'intervalle de confiance à $95 \%$ est rapporté. Les statistiques ont été réalisées avec le logiciel MedCalc version 13.1.0 (MedCalc Software bvba, Ostend, Belgium ; http://www.medcalc.org ; 2014). Une valeur de $\mathrm{p}<0,05$ était considérée comme statistiquement significative.

\section{RÉSULTATS}

Trente sujets ont été inclus (50\% d'hommes). L'âge moyen était de $20+/-1$ ans. Quatre sujets (13\%) étaient fumeurs. L'indice de masse corporelle était de 21,66 +/- 2,06 kg/m².

Le SDV moyen était de 0,50 +/- 3,70 lors de la première mesure. L'erreur typique de mesure 
est de 1,98 pour le SDV, 1,51 pour le score AGS, 0,86 pour le score F-L, 0,48 pour le score AGMI et 0,83 pour le score AGPI oméga 3. Les autres résultats nutritionnels ainsi que les ICCs sont présentés dans le tableau 1. Le délai moyen entre les 2 mesures était de $19+/-9$ jours.

\section{DISCUSSION}

Notre étude met en évidence que la reproductibilité du questionnaire est satisfaisante dans cette population étudiante. De plus, les sujets de cette population ont une alimentation défavorable montrant l'intérêt de réaliser ce type d'évaluation.

Des études précédentes réalisées avec ce questionnaire avaient mis en évidence qu'un SDV inférieur ou égal à moins 1 était retrouvé chez les patients coronariens, chez les patients avec une artériopathie des membres inférieurs et chez des patients ayant présenté un accident vasculaire cérébral ischémique. Un SDV inférieur à moins 1 a ainsi été considéré comme une alimentation à risque vasculaire Un SDV de +8 était retrouvé chez des patients ayant reçu des conseils alimentaires dans un centre de réhabilitation cardio-vasculaire . Ainsi un SDV supérieur ou égal à 8 a été suggéré comme optimal. Finalement un SDV inférieur à 8 a été considéré comme une alimentation qui méritait d'être améliorée pour limiter le risque vasculaire. Il est à noter que malheureusement, à ce jour, ce questionnaire n'a pas été utilisé dans une étude de suivi pour essayer de définir le risque en fonction de la valeur de SDV. Les données que nous avons sont seulement liées et extrapolées d'études transversales.

Le SDV moyen de 0,50+/- 3,70 retrouvé lors de la première mesure met en évidence une alimentation qui est proche du SDV (-1) retrouvé chez les patients présentant une maladie athéromateuse. De plus ce SDV est plus défavorable que le SDV moyen retrouvé $(3,30+/-$ 4,80) dans une étude de 250 sujets âgés de 50 ans en moyenne. Dans cette population de médecine générale, le questionnaire alimentaire était proposé à toutes les personnes venant 
consulter. Ce résultat confirme les résultats précédents qui suggéraient que l'alimentation était moins favorable chez les sujets jeunes . Il a été suggéré que le caractère moins favorable de l'alimentation des sujets jeunes comparativement aux sujets plus âgés est lié à une consommation alimentaire différente comme davantage de grignotage et une conscience de la santé moindre . De plus les patients présentant des pathologies chroniques sont probablement plus réceptifs aux différentes possibilités «thérapeutiques » (avoir une meilleure alimentation) qui peuvent être suggérées par leur médecin pour améliorer leur santé.

L'analyse des différents groupes alimentaires dans cette population d'étudiants met en évidence comparativement aux sujets d'une population issue de médecine générale, une tendance à une consommation moindre de fruits et légumes $(3,53+/-1,59$ vs 4,60 +/- 1,70), une tendance à une consommation plus importante d'acides mono-insaturés $(1,20+/-1,00 v \mathrm{~s}$ $1,10+/-0,90)$, une tendance à une consommation moindre d'acides gras poly-insaturés oméga $3(1,57+/-1,65$ vs $2,10+/-1,70)$ mais une tendance à une consommation plus importante d'acides gras saturés $(5,73+/-2,70$ vs 4,5+/-2,60). Ces résultats mettent ainsi en évidence que l'alimentation des étudiants est loin d'être optimale et soulignent l'intérêt d'agir à ce niveau.

La reproductibilité du SDV et des autres scores est satisfaisante au regard des résultats de l'erreur typique de mesure et des comparaisons entre la mesure 1 et la mesure 2. Les ICC vont de 0,67 pour le score F-L et à 0,76 pour le score AGPI omega 3. Ces résultats sont globalement mois bons que ceux retrouvés par Laviolle et al. qui retrouvaient des ICC allant de 0,71 pour le score d'acides gras mono-insaturés à 0,93 pour le SDV. Ces différences peuvent s'expliquer par un plus grand délai entre l'administration des 2 questionnaires dans notre population $(19+/-9$ jours $)$ comparé à celui retrouvé dans la population de Laviolle et al. (16+/- 3 jours). D'ailleurs, il se trouve que la plus grande différence entre le SDV (mesure 1) et le SDV (mesure 2) égale à 7, est retrouvée chez un sujet dont le délai entre les deux mesures était de 44 jours. De plus une alimentation moins « réglée » de trois repas par jour 
chez les étudiants que chez les personnes plus âgées pourrait être une explication. Malheureusement, ce travail ne permet pas d'étudier ce paramètre puisqu'aucune question sur le nombre de repas par jour ou sur la régularité de l'alimentation n'était posée.

\section{Limites}

La principale limite de ce travail est le délai important entre la $1^{\text {ère }}$ mesure et la $2^{\text {ème }}$ mesure. En effet, nous n'avons pas pu organiser ces différentes mesures dans un délai plus court et identique pour l'ensemble des étudiants. De plus, nous n'avons pas recueilli les différentes activités sportives réalisées par ces étudiants et il n'est pas possible d'exclure que la participation de certains étudiants à des compétitions sportives ait pu modifié leur alimentation entre les deux mesures. Un autre élément à prendre en compte est que ce questionnaire ne prend pas en compte le nombre de repas et le grignotage réalisés par les sujets. De plus, il est important de souligner que cette relativement bonne reproductibilité retrouvée a été mise en évidence chez des étudiants sportifs faisant possiblement davantage attention à leur alimentation que des étudiants lambda. Cependant, l'objectif de ce travail était focalisé sur ce type de population puisque nous comptons étudier par la suite le lien entre l'activité physique de ces étudiants et leur alimentation.

\section{CONCLUSION}

Ce court questionnaire validé (14-item FFQ) possède une bonne reproductibilité $(\mathrm{ICC}=0,68)$ chez les étudiants. La valeur d'ICC est néanmoins moins élevée que lors de l'étude de validation dans une population de patients $(\mathrm{ICC}=0,93)$. Ce travail confirme que ce questionnaire peut être utilisé dans de plus larges populations étudiantes afin d'évaluer l'alimentation à risque vasculaire. 
Remerciement : Les auteurs remercient l'Université Catholique de l'Ouest (UCO) qui a financé le projet.

\section{Conflit d'intérêt : aucun}

\section{TABLEAUX}

Tableau 1 : Scores nutritionnels et reproductibilités dans la population étudiée évalués par le questionnaire nutritionnel.

\begin{tabular}{lccc|c|c} 
& \multicolumn{2}{c|}{ Questionnaire nutritionnel } & $P$ & $\begin{array}{c}\text { Intra Classe } \\
\text { Coefficient (ICC) }\end{array}$ & $\begin{array}{c}\text { Erreur typique } \\
\text { de mesure }\end{array}$ \\
\hline $\begin{array}{l}\text { Scores } \\
\text { AGS } \\
(0 \text { à 17 points })\end{array}$ & $5,73+/-2,70$ & $5,53+/-2,79$ & NS & $0,71[0,47-0,85]$ & $1,51[1,21-2,03]$ \\
$\begin{array}{l}\text { AGMI }(0 \text { à } 4 \\
\text { points })\end{array}$ & $1,20+/-1,00$ & $1,33+/-0,80$ & NS & $0,73[0,50-0,86]$ & $0,48[0,38-0,55]$ \\
$\begin{array}{l}\text { AGPI } \Omega-3(0 \text { à } \\
8 \text { points })\end{array}$ & $1,57+/-1,65$ & $1,40+/-1,69$ & NS & $0,76[0,56-0,88]$ & $0,57[0,46-0,77]$ \\
$\begin{array}{l}\text { F-L }(0 \text { à } 7 \\
\text { points })\end{array}$ & $3,53+/-1,59$ & $3,30+/-1,37$ & NS & $0,67[0,47-0,81]$ & $0,86[0,71-1,11]$ \\
$\begin{array}{l}\text { SDV }(-17 \text { à }+19 \\
\text { points })\end{array}$ & $0,50+/-3,70$ & $0,30+/-3,14$ & NS & $0,68[0,43-0,83]$ & $1,98[1,58-2,67]$ \\
\hline
\end{tabular}

Tableau 1 légende : Les résultats sont exprimés en moyenne +/- écartype. AGS = acides gras saturés, AGMI = acides gras mono-insaturés, AGPI $\Omega-3=$ acides gras poly-insaturés omega $3, \mathrm{~F}-\mathrm{L}=$ fruits et légumes et $\mathrm{SDV}=$ score diététique vasculaire. $\mathrm{NS}=$ non significatif. 


\section{REFERENCES :}

1.Leal J, Luengo-Fernandez R, Gray A, Petersen S and Rayner M. Economic burden of cardiovascular diseases in the enlarged European Union. Eur Heart J. 2006;27(13):1610-9.

2.Iqbal R, Anand S, Ounpuu S, Islam S, Zhang X, Rangarajan S, et al. Dietary patterns and the risk of acute myocardial infarction in 52 countries: results of the INTERHEART study. Circulation. 2008;118(19):1929-37.

3.Nordmann AJ, Suter-Zimmermann K, Bucher HC, Shai I, Tuttle KR, Estruch R, et al. Meta-analysis comparing Mediterranean to low-fat diets for modification of cardiovascular risk factors. The American journal of medicine. 2011;124(9):841-51 e2.

4.Sattelmair J, Pertman J, Ding EL, Kohl HW, 3rd, Haskell W and Lee IM. Dose response between physical activity and risk of coronary heart disease: a meta-analysis. Circulation. 2011;124(7):789-95.

5.Pahkala K, Hietalampi H, Laitinen TT, Viikari JS, Ronnemaa T, Niinikoski H, et al. Ideal cardiovascular health in adolescence: effect of lifestyle intervention and association with vascular intima-media thickness and elasticity (the Special Turku Coronary Risk Factor Intervention Project for Children [STRIP] study). Circulation. 2013;127(21):2088-96.

6.Aatola H, Hutri-Kahonen N, Juonala M, Laitinen TT, Pahkala K, Mikkila V, et al. Prospective relationship of change in ideal cardiovascular health status and arterial stiffness: the Cardiovascular Risk in Young Finns Study. Journal of the American Heart Association. 2014;3(2):e000532.

7.Laviolle B, Froger-Bompas C, Guillo P, Sevestre A, Letellier C, Pouchard M, et al. Relative validity and reproducibility of a 14-item semi-quantitative food frequency questionnaire for cardiovascular prevention. Eur J Cardiovasc Prev Rehabil. 2005;12(6):587-95.

8.Carsin $\mathrm{M}$ and Mahe G. [Why should vascular patients have a dietary assessment?]. J Mal Vasc. 2010;35(1):17-22.Pourquoi devons-nous evaluer l'alimentation des sujets a risque vasculaire?

9.Carsin-Mahe M, Abraham P, Le Faucheur A, Leftheriotis G and Mahe G. Simple routine assessment of dietary pattern in patients with peripheral artery disease. J Vasc Surg. 2012;56(1):281-2.

10.Mahe G. Regarding "Nutrition impacts the prevalence of peripheral arterial disease in the United States". J Vasc Surg. 2009;49(6):1632; author reply 32.

11.Mahe G, Ronziere T, Laviolle B, Golfier V, Cochery T, De Bray JM, et al. An unfavorable dietary pattern is associated with symptomatic ischemic stroke and carotid atherosclerosis. J Vasc Surg. 2010;52(1):62-8.

12.Froger-Bompas C, Laviolle B, Guillo P, Letellier C, Ligier K, Daubert JC, et al. Sustained positive impact of a coronary rehabilitation programme on adherence to dietary recommendations. Arch Cardiovasc Dis. 2009;102(2):97-104.

13.Mahe G, Carsin M, Zeeny M and De Bosschere JP. Dietary pattern, a modifiable risk factor that can be easily assessed for atherosclerosis vascular disease prevention in clinical practice. Public Health Nutr. 2011;14(2):319-26.

14. Volatier J. Enquête individuelle et nationale sur les consommations alimentaires. . Paris: TEC \& DOC; 2000.

15.Hopkins W. Measures of reliability in sports medicine and science. Sports Medicine. 2000;30(1):115.

16.Landis JR and Koch GG. The measurement of observer agreement for categorical data. Biometrics. 1977;33(1):159-74.

17.Smith KJ, McNaughton SA, Gall SL, Blizzard L, Dwyer T and Venn AJ. Takeaway food consumption and its associations with diet quality and abdominal obesity: a cross-sectional study of young adults. The international journal of behavioral nutrition and physical activity. 2009;6:29.

18.Ribas-Barba L, Serra-Majem L, Salvador G, Castell C, Cabezas C, Salleras L, et al. Trends in dietary habits and food consumption in Catalonia, Spain (1992-2003). Public Health Nutr. 2007;10(11A):1340-53.

19.Johansson L, Thelle DS, Solvoll K, Bjorneboe GE and Drevon CA. Healthy dietary habits in relation to social determinants and lifestyle factors. Br J Nutr. 1999;81(3):211-20. 
Questionnaire alimentaire (14-item Food-Frequency Questionnaire).

\section{Questionnaire alimentaire}

1. Consommez-vous du fromage ? ( 1 portion $=1 / 8^{\text {ème }}$ de camembert $=30 \mathrm{~g}$ ) moins de 2 portions par semaine

3 à 6 portions par semaine

1 portion par jour

2 portions par jour

3 portions par jour ou plus

2. Consommez-vous de la viande? (hors volailles)
0 à 2 fois par semaine
3 à 6 fois par semaine
7 fois par semaine ou plus

3. Consommez-vous du poisson (frais, surgelé, ou en conserve) ?
$\square \quad$ moins d'une fois par semaine
1 fois par semaine
2 à 3 fois par semaine
4 fois par semaine ou plus

4. Consommez-vous de la charcuterie (y compris saucisses, cassoulet, choucroute) sauf le jambon dégraissé ?
0 à 1 fois par semaine
2 à 3 fois par semaine
4 à 6 fois par semaine
7 fois par semaine ou plus

5. Consommez-vous des quiches, tartes salées, pizzas, friands, sandwichs du commerce ?

$\square \quad 0$ à 1 fois par semaine

2 à 3 fois par semaine

4 fois par semaine ou plus

6. Consommez-vous des frites ?

fréquence : par semaine

frites « maison » à l'huile, type d'huile :

frites « maison » à la végétaline

frites au four surgelées

restaurant ou self

7. Consommez-vous des viennoiseries (croissants, brioches etc...), ou des pâtisseries du commerce?

0 à 1 fois par semaine

2 à 4 fois par semaine

5 fois par semaine ou plus

8. Consommez-vous des fruits et pur jus de fruits : 1 portion = 1 fruit moyen = 1verre de jus de fruit $(200 \mathrm{ml})$
$\square \quad 0$ à 2 portions par semaine
3 à 6 portions par semaine
7 à 13 portions par semaine (au moins 1 fruit par jour)
14 portions par semaine ou plus (au moins 2 fruits par jour)

9. Actuellement, consommez-vous des noix ?

oui, quantité par jour

non

10. Consommez-vous des légumes cuits et soupe de légumes : 1 portion = 1 assiette ou 1 bol 
0 à 2 portions par semaine

3 à 7 portions par semaine

8 portions ou plus par semaine

11. Consommez-vous des crudités ou salades?
0 à 2 portions par semaine
3 à 7 portions par semaine
8 portions par semaine ou plus

12. Consommez-vous du beurre ? (et crème fraîche) : 1 portion = 1 plaquette individuelle de 10 à $15 \mathrm{~g}$ jamais

cru, 1 portion par jour

cru, 2 portions par jour

$\mathrm{cru}, 3$ portions par jour

cru et utilisé en cuisine (soit plus de 3 portions par jour)

13. En dehors du beurre, utilisez-vous une autre matière grasse ? (type margarine)

- pour cuisiner?

non

oui, laquelle

- à 1 repas par jour (environ 1 plaquette individuelle)

- à 2 repas par jour (environ 2 plaquettes individuelles)

- pour tartiner, assaisonner vos plats chauds?

non

oui, laquelle

- à 1 repas par jour (soit 1 plaquette individuelle)

- à 2 repas par jour (soit 2 plaquettes individuelles)

- 3 portions ou plus par jour (plus de 3 plaquettes individuelles)

14. Consommez-vous de l'huile?

- pour cuisiner?

$\square$ non

$\square \quad$ oui, laquelle?

- à 1 repas par jour (environ 1 cuillère à soupe)

pour vos vinaigrettes?

- à 2 repas par jour (environ 2 cuillères à soupe)

$\square$ non

oui, laquelle?

- 1 assaisonnement par jour (environ 1 cuillère à soupe)

- 2 assaisonnements par jour (environ 2 cuillères à soupe)

- 3 assaisonnements ou plus (plus de 2 cuillères à soupe) 